\title{
Congenital anomalies of the aortic arch
}

\author{
Sarv Priya ${ }^{1}$, Richard Thomas ${ }^{1}$, Prashant Nagpal $^{2}$, Arun Sharma ${ }^{3}$, Michael Steigner ${ }^{1}$ \\ ${ }^{1}$ Division of Non-Invasive Cardiovascular Imaging, Department of Radiology, Brigham and Women's Hospital, Harvard Medical School, Boston, \\ MA, USA; ${ }^{2}$ Department of Radiology, University of Iowa Hospitals and Clinics, Iowa, USA; ${ }^{3}$ Department of Cardiovascular Radiology, All India \\ Institute of Medical Sciences, New Delhi, India \\ Contributions: (I) Conception and design: S Priya, R Thomas, M Steigner; (II) Administrative support: S Priya, R Thomas, M Steigner; (III) Provision \\ of study material or patients: All authors; (IV) Collection and assembly of data: All authous; (V) Data analysis and interpretation: All authors; (VI) \\ Manuscript writing: All authors; (VII) Final approval of manuscript: All authors. \\ Correspondence to: Sarv Priya, MD. Division of Non-Invasive Cardiovascular Imaging, Department of Radiology, Brigham and Women's Hospital, \\ Harvard Medical School, Boston, MA, USA. Email: sarvpriya1985@gmail.com.
}

\begin{abstract}
Congenital anomalies of the aortic arch include diverse subgroups of malformations that may be clinically silent or may present with severe respiratory or esophageal symptoms especially when associated with complete vascular rings. These anomalies may be isolated or may be associated with other congenital heart diseases. Volume rendered computed tomography (CT) and magnetic resonance angiography (MRA) help in preoperative surgical planning by providing information about the complex relationship of aortic arch and its branches to the trachea and esophagus. Three dimensional capabilities of both computed tomography angiography (CTA) and MRA are helpful in determining evidence of tracheal or esophageal compression or other high-risk features in patients with a complete vascular ring.
\end{abstract}

Keywords: Aortic arch; vascular ring; computed tomography angiography (CTA); magnetic resonance angiography (MRA)

Submitted Sep 10, 2017. Accepted for publication Oct 17, 2017.

doi: $10.21037 / \mathrm{cdt} .2017 .10 .15$

View this article at: http://dx.doi.org/10.21037/cdt.2017.10.15

\section{Introduction}

Congenital aortic arch malformations present a large spectrum of variations and anomalies that emanate from disordered embryogenesis of branchial arches. These aberrations are the result of either abnormal persistence or involution of embryonic vascular segments. Clinically, these malformations can be asymptomatic in terms of their detection especially when imaging is being performed for some other reasons. However, these can be symptomatic in the form of difficulty in breathing or swallowing. These malformations can be isolated or they may be associated with other intracardiac and/or chromosomal defects. Imaging plays a great role in the detection of these anomalies and thus helps much in accurate preoperative surgical decisions. The primary role of a radiologist is to detect these abnormalities and, importantly, to look for imaging features which indicate high risk of compression of the trachea and esophagus.

Historically, barium swallow study was the primary mode of investigation to look for evidence of indentations on the posterior wall of esophagus as seen on lateral view. A negative esophagram may rule out the presence of vascular ring. However, even if it is positive, this still would not depict the type of vascular anomaly. The upper gastrointestinal study also is relatively difficult to perform in pediatric patients. All these factors led to the need of invasive modalities like angiography for surgical planning. Currently, non-invasive multidetector three-dimensional (3D) CT and 3D MRA play a greater role in the detection and characterization of these anomalies. These techniques also provide a more detailed evaluation of the tracheoesophageal anatomy which is not delineated clearly by the $2 \mathrm{D}$ imaging modalities like conventional radiography, esophagram and angiography. However, an exact knowledge of anatomy is essential to avoid unnecessary surgical 
complications.

In this review, we proceed to discuss various aortic arch malformations along with their associated aberrations. We also take stock of surgical parameters that influence various management strategies. And most importantly, we discuss how to properly approach a case with suspected aortic arch pathology.

\section{Imaging}

Various imaging modalities are available, and each has its own merits and demerits. The use of conventional modalities, including barium esophagram, have fallen presently out of place because of acute difficulty in performing them, especially in pediatric patients. Another reason for not using them is that they are able to provide 2D information only. Catheter angiography, another 2D technique, has also lost its charm due to the advent of other non-invasive imaging modalities. Echocardiography is often used as the first imaging modality, especially in pediatric patients. However, it is operator-dependent, and as such, is challenging in evaluation of complex arch anomalies. Both computed tomography (CT) and magnetic resonance imaging (MRA) are therefore indispensable for accurate description of pre-surgical anatomy.

CT provides high spatial resolution evaluation of vascular anomalies and can be reformatted in multiple oblique planes to provide a complete assessment of the tracheal and esophageal compression simultaneously. With the availability of new generation scanners that have advanced radiation dose reduction technologies, the radiation dose has reduced dramatically. CT is usually performed without use of gating which further reduces the exposure. High pitch spiral mode can be used without gating to drastically reduce cardiac pulsation artifacts (1). This may favor its increasing use, especially in pediatric patients, as CT may be performed without use of anesthesia or breath-holding (2).

MRI is an excellent multiplanar imaging modality that provides evaluation of aortic arch malformations without radiation exposure. Black blood and bright blood-both non-contrast-sequences as well as contrast enhanced MRA can be employed and are cardiac gated to reduce motion artifacts. Three dimensional capabilities provide anatomic aortic arch relationships as well as the assessment of tracheal compression. Unfortunately, MRI is generally time consuming and it also requires general anesthesia in infants and most of the young children $(3,4)$. However, the availability of new techniques including compressed sensing, 3D ultra-shot echo time and 4D flow acquisition result in a significant reduction of scan time. These techniques make free breathing MRI highly feasible with little or no requirement of sedation (5-7). 4D contrast-enhanced MRA with k-space undersampling techniques such as keyhole method results in shorter scan times and is more effective in imaging pediatric patients with rapid heart rates (8). The diagnostic quality is also acceptable with minimum motion artifacts. The availability of these techniques is, however, still very limited.

Recently there has been raised a concern regarding the deposition of gadolinium based contrast agents on repeat administration of gadolinium in deep gray matter of the brain, predominantly the dentate nuclei and globus pallidus. However, the clinical significance of this is still not clear $(9,10)$. As a result of this recent discovery, there has been an increasing demand for non-contrast enhanced MRA especially for the patients who require multiple follow up studies. Unenhanced MRA including 3D ECG gated and respiratory navigated balanced steady-state free precision (SSFP) techniques obviate the need of contrast in these patients and the image quality here is also more acceptable as compared to contrast enhanced MRA (11-14). The patients with renal failure may also benefit from unenhanced MRA since they cannot receive gadolinium due to risk of nephrogenic systemic fibrosis.

The decision to choose the imaging modality is primarily based on the available facilities as well as the imaging teams' expertise. The merits and demerits of various imaging modalities are given in Table 1 .

\section{Embryology of aortic arch development and anomalies}

An exact understanding of the development of aortic arch is essential in order to classify and interpret various aortic arch malformations. The development of branchial arches begins by the second week of gestation and continues till the seventh week. There are six paired arches (numbered cranial-caudally) that connect the paired dorsal and ventral aorta. This model was originally described by Rathke as represented in classical Rathke's diagram (Figure 1). All of these arches are not present at the same time; rather, they appear and undergo regression sequentially from cranial to caudal direction. The first, second, and the fifth arches involute. The remnants of the first and second arch form maxillary and stapedial arteries respectively (15). The fifth 
Table 1 Merits and demerits of different diagnostic modalities

\begin{tabular}{|c|c|c|}
\hline Modality & Advantages & Disadvantages \\
\hline $\begin{array}{l}\text { Conventional } \\
\text { angiography }\end{array}$ & $\begin{array}{l}\text { High spatial resolution, ability to demonstrate complex } \\
\text { connections and provide real time information, ability } \\
\text { to grade stenosis using pressure gradient information }\end{array}$ & $\begin{array}{l}\text { Invasive modality, need for iodinated contrast material, inability } \\
\text { to provide 3D information, information provided is limited to } \\
\text { the lumen and one cannot evaluate associated anomalies }\end{array}$ \\
\hline $\begin{array}{l}\text { Magnetic } \\
\text { resonance } \\
\text { angiography }\end{array}$ & $\begin{array}{l}\text { Ability to provide multiplanar and 3D images, ability } \\
\text { to provide extraluminal information with excellent soft } \\
\text { tissue contrast, ability to provide information without } \\
\text { use of contrast material }\end{array}$ & $\begin{array}{l}\text { Expensive, limited availability, time consuming modality which } \\
\text { may limit use in sick patients or may warrant sedation, images } \\
\text { can be degraded by respiratory motion and often requires } \\
\text { patient's cooperation, cannot be used in claustrophobic } \\
\text { patients or those with contraindications like metallic foreign } \\
\text { body, etc., non-contrast MRA may be degraded by artifacts } \\
\text { and often provides limited information }\end{array}$ \\
\hline
\end{tabular}

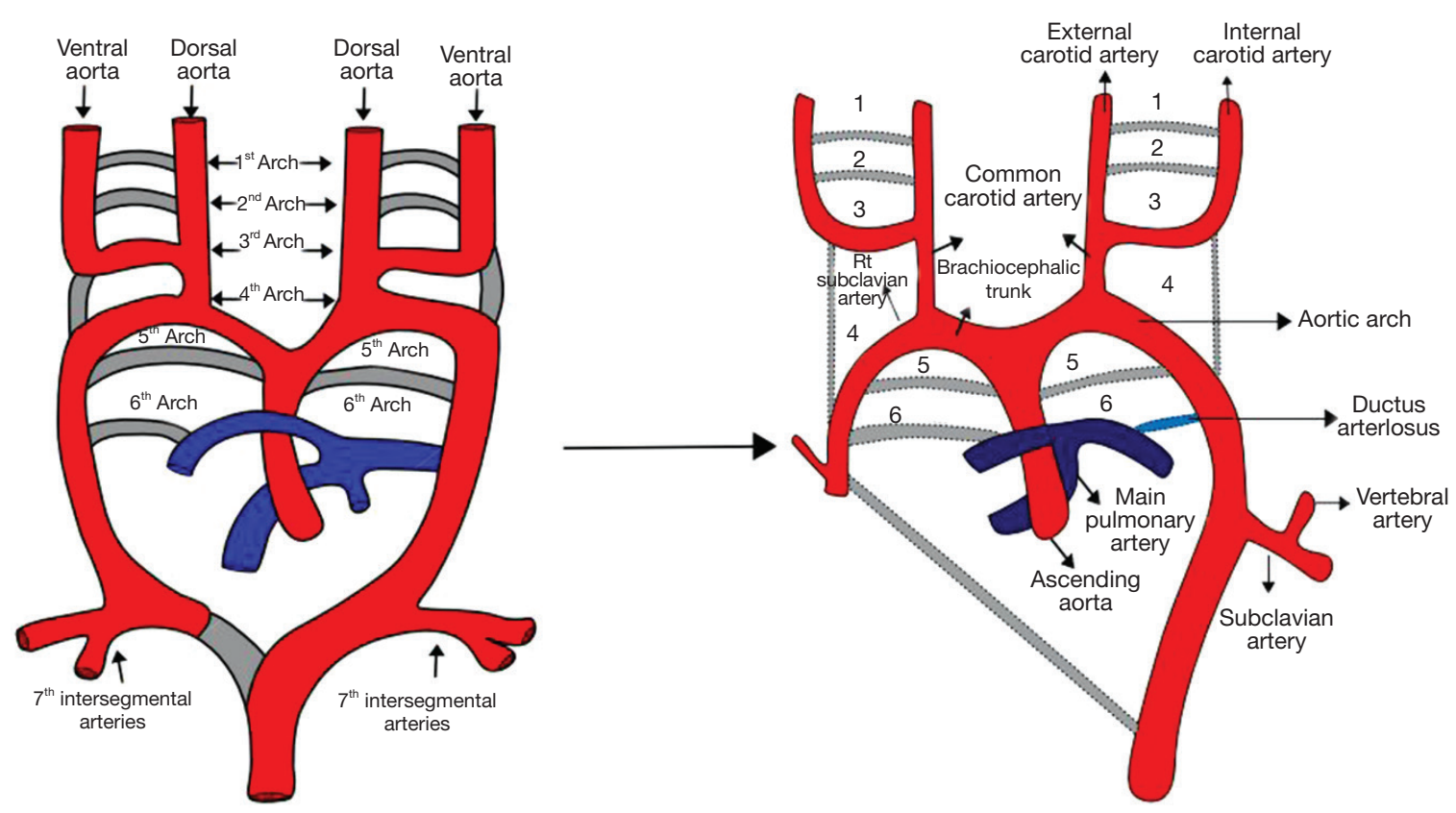

Figure 1 Rathke's diagram, schematic representation of the development of the aortic arch and branches. Definitive aortic arch is formed from the fourth arch, while the pulmonary artery is formed from the anterior bud of the sixth arch. Parts of the third arch and anterior segments of the branchial arches contribute to formation of left common, external and internal carotid arteries. Areas that are shown in red persist where as those shown in grey eventually disappear. 
arch may rarely remain patent which explains the double barrel aorta. The principal arches that form the aortic branches are the third, fourth and the sixth. The third arch forms common carotid and a segment of internal carotid arteries while the fourth arch forms the definitive aortic arch. The ventral portion of the sixth arch forms the distal segments of pulmonary artery whereas the dorsal portion of the arch forms the ductii arteriosus. The ventral aorta forms truncus arteriosus which later divides into ascending aorta and proximal segment of main pulmonary artery. The paired dorsal aorta fuses to form a single trunk. The seventh intersegmental artery (branch of dorsal aorta) forms a part of the subclavian arteries (16).

In order to classify and understand the morphology of various subtypes of aortic arch anomalies and variants, it is important to understand another model that was proposed by Edwards. He proposed a hypothetical developmental model that consisted of paired aortic arches on either side, paired bilateral ductus arteriosus and a single dorsal aorta. The carotid and subclavian vessels here arise from the aortic arch. The carotid vessels are anteriorly located where as the subclavian arteries are posteriorly located.

Normal left aortic arch is formed by the regression of the right aortic arch, right sided ductus and right dorsal aorta. The proximal portion of right dorsal aorta forms part of right subclavian artery. The left dorsal aorta forms the distal aortic arch and the descending thoracic aorta $(17,18)$.

The various anomalies in aortic arch can be explained by the pattern of breaks at different levels in the Edwards' double aortic arch model. Abnormal persistence or regression of various segments leads to several types of malformations.

\section{Aortic arch variations and anomalies}

\section{Classification}

The prevalence of aortic arch malformations varies between $1-2 \%$ among the general population (19). There are several ways to classify these malformations which are based either on anatomical features like arch sidedness, course and location of arch, interruption, persistent fifth arch or on the functional outcome of features like respiratory compromise or esophageal dysfunction (20). Some authors have also classified these malformations as obstructive or non-obstructive aortic arch anomalies with observations that obstructive anomalies are more common in pediatric populations (21). Most of these can be explained by Edwards's hypothetical double arch system as explained before (22).

In this review we will follow an imaging approach that helps in defining the type of abnormality and also in identifying the salient high-risk imaging features which may result in respiratory distress or dysphagia. We would also emphasize on the close mimickers of these anomalies and find out ways to differentiate them.

\section{Arch sidedness and branching pattern}

The imaging report should begin with a description of the arch sidedness. It is described on the basis of right or left location of the aortic arch with respect to trachea and its course in relation to the main bronchus. In the normal branching pattern, the first branch (innominate or brachiocephalic artery) usually travels to the side opposite to that of the aortic arch. There are, of course, a few exceptions like the retroesophageal innominate artery that instead of being the first arises as the last branch from the aortic arch. In some patients, the origin of both carotid arteries may be close to each other, and thus making it difficult to determine about the first branch (21).

\section{Left aortic arch}

Left aortic arch lies on the left of trachea and courses over the left main bronchus. The structures that involute in the formation of normal left arch include distal segment of the right fourth branchial arch lying between the right subclavian artery and the descending thoracic aorta, right sided dorsal aorta distal to the seventh intersegmental artery origin and the right sided ductus arteriosus. The persistence of left fourth branchial arch forms the definitive aortic arch (18). The ductus remains patent on the left side and connects the aorta distal to the left subclavian artery origin to the proximal left pulmonary artery. The descending aorta is usually on the same side as is the aortic arch, and it is due to the persistence of ipsilateral dorsal aorta (23). The exact mechanism of the predominant sidedness of the arch is still unknown.

\section{Normal left aortic arch branching pattern}

In the normal branching pattern, the first branch is the right innominate artery followed by the left common carotid and finally the left subclavian artery (Figures 2,3 ). The right brachiocephalic (or the innominate) artery further divides into right common carotid and right subclavian arteries (16). 


\section{Normal variations in aortic arch branching pattern}

\section{Bovine arch-A mistaken identity}

The most common left aortic arch branching variation

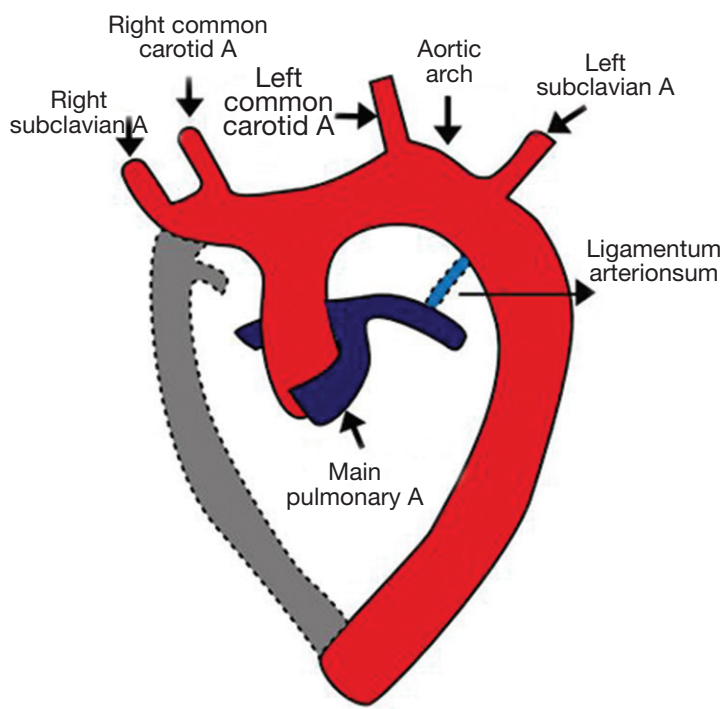

Figure 2 Schematic representation of the normal branching pattern of the left aortic arch. The first branch is right innominate artery giving rise to right common carotid and right subclavian artery, followed by the left common carotid and left subclavian artery. Ductus is usually on the left side persisting as ligamentum arteriosus. is often incorrectly labeled as the bovine aortic arch. The reported incidence varies in between $10-20 \%$ of the population. The bovine arch seen in cattle has the origin of all three branches from a single trunk. However, in the normal variation, the left common carotid artery either shares generally a common origin or, less commonly, arises directly from the right innominate artery (Figure 4). The left subclavian artery arises separately from the arch (24). Initially thought to be a normal anatomic variant, the bovine aortic arch has recently been linked with the development of thoracic aneurysms with, of course, an increased risk of torsion and deceleration injuries. This risk is due to the presence of only two fixation points in place of usual three points $(25,26)$. This anomaly is no longer considered a clinically insignificant variant since it is associated with a high incidence of thoracic aortic disease and its presentation comparatively in a younger age (27).

\section{Vertebral artery origin variations}

The other often encountered variation is the origin of left vertebral artery directly from the aortic arch with a prevalence of $2.4-5.8 \%$ in autopsy series (28) (Figure 5). However, the aberrant origin of right vertebral artery is more rare (29). There may also be fenestration or duplication of vertebral artery origins. These variations may have no clinical significance, but they should be documented as they may have certain surgical implications (30).
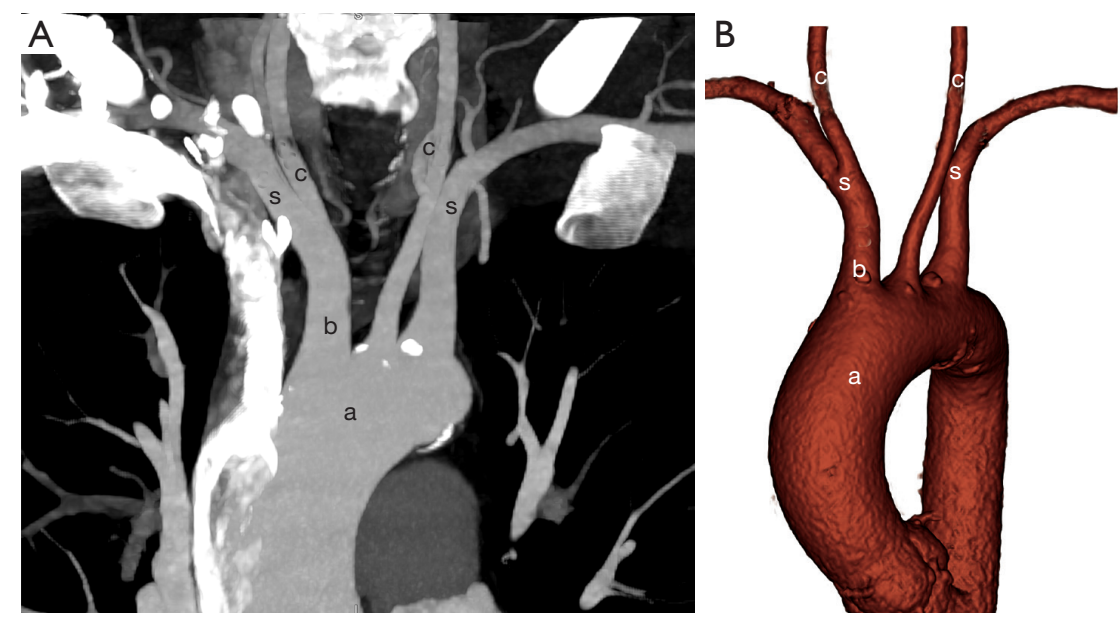

Figure 3 Left aortic arch with normal branching pattern: a 54-year-old male with acute chest pain, CTA to rule out dissection. Coronal CTA maximum intensity projection (MIP) (A) and 3D (B) images show a left aortic arch with (a) normal branching pattern. Note brachiocephalic artery (b), common carotid arteries (c) and subclavian arteries (s). 


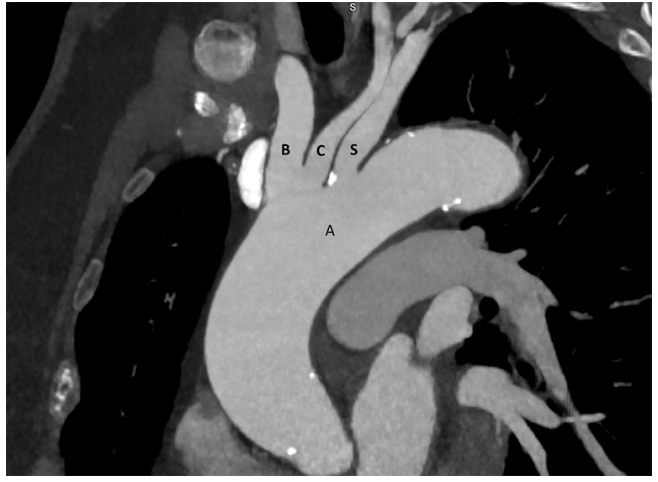

Figure 4 Common origin of brachiocephalic trunk and left common carotid artery: a 65-year-old male on follow up for known ascending aortic aneurysm. Oblique MIP CTA shows common origin of brachiocephalic trunk (B) and left common carotid artery (C) from a left aortic arch (A). Note left subclavian artery (S).

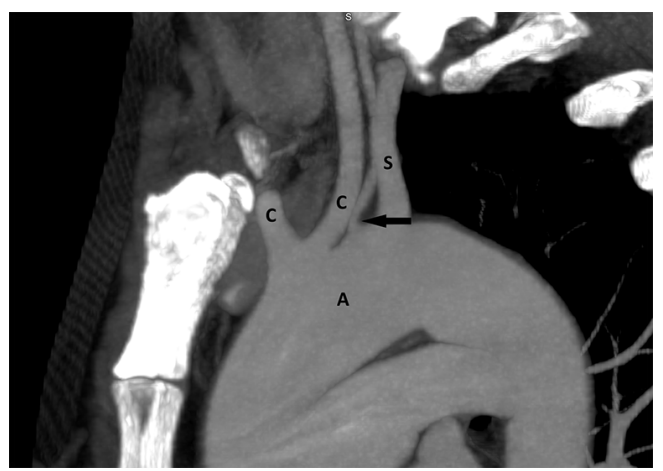

Figure 5 Direct origin of the left vertebral artery from the aortic arch: CTA to evaluate aortic dilatation seen on chest radiograph in a 69-year-old female with chest pain. Oblique sagittal CTA MIP image shows direct origin of the left vertebral artery (arrow) from the aortic arch (A). Note left subclavian artery (S) and bilateral common carotid arteries $(\mathrm{C})$.

\section{Left aortic arch with aberrant right subclavian artery (ARSA)}

ARSA is the most common anomaly associated with left aortic arch and is predominant in females (31). This anomaly results from involution of right arch between the right subclavian and the right common carotid artery along with regression of the right ductus arteriosus. The proximal segment of the ARSA arises from the distal right dorsal aorta instead of arising from the right fourth arch and follows a retroesophageal course (32). This anomaly does not result in complete vascular ring as there is nothing on the right side of trachea. However, it may

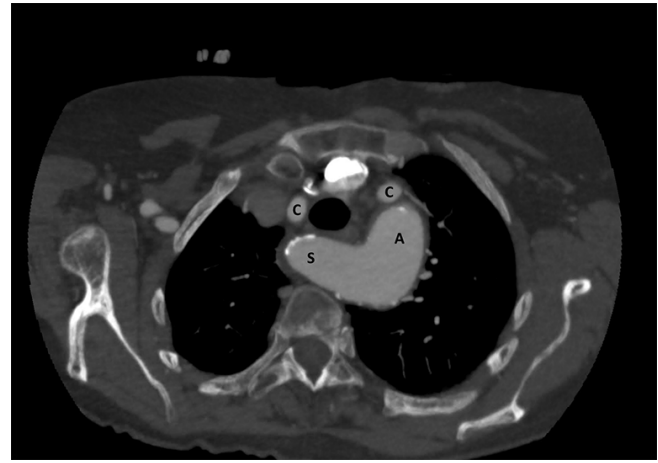

Figure 6 Aberrant right subclavian artery with diverticulum of Kommerell: a 51-year-old male with recurrent strokes, CTA to evaluate aortic arch and its branches. Axial CTA shows aberrant origin of the right subclavian artery $(\mathrm{S})$ with dilatation at its origin, called diverticulum of Kommerell. The aortic arch (A) and both common carotid arteries (C) are visualized.

result in a syndrome called "dysphagia lusoria", that is, esophageal compression, and it happens due to an aberrant retroesophageal course. The most common course of the aberrant vessel is retroesophageal. However, it may course between trachea and esophagus or even anterior to the trachea (33). It may also be associated with other congenital anomalies including patent ductus arteriosus (PDA), ventricular septal defect or coarctation of aorta. The association between the aberrant subclavian artery and the bicarotid trunk (common origin of the both common carotid arteries) is more symptomatic than the aberrant course alone (34). The salient feature to document is the presence or absence of diverticulum of Kommerell. The presence of diverticulum is a high-risk feature especially if ectatic or aneurysmal. The anomaly does not require an intervention as it is usually an incidental finding and is asymptomatic as well. In patients without diverticulum the ductus is usually ipsilateral to the arch thus no formation of a vascular ring.

\section{ARSA with diverticulum of Kommerell}

The retroesophageal diverticulum arises because of the persistence of segment of the right sixth arch (Figure 6). When the aberrant subclavian artery arises from the Kommerell diverticulum, the ductus completing the vascular ring is usually on the opposite side of the arch (35). In patients with aneurysm of the diverticulum of Kommerell, it may be surgically resected. 


\section{Left aortic arch with isolation of subclavian artery}

In this rare anomaly, the subclavian artery has no connection with the aortic arch. This unusual malformation is the result of some involution in right arch at two levels: one is between the left subclavian and the left common carotid artery, and the other is distal to the right ductus and right subclavian artery. The subclavian artery is reconstituted from either the vertebral artery or the ductus arteriosus (36). This should not be confused with an interrupted aortic arch (IAA) in which discontinuity is actually of the descending aorta itself. This anomaly may be associated with some other congenital cardiovascular diseases, such as PDA, tetralogy of Fallot or chromosomal aberrations in the nature of $22 \mathrm{q} 11$ deletion syndrome which has been described in syndromic anomalies like CHARGE (coloboma, heart anomalies, choanal atresia, retardation of growth and development, and genital and ear anomalies) syndrome $(37,38)$. This anomaly does not constitute a vascular ring. Clinically, these patients are asymptomatic though sometimes may have features of left upper limb ischemic pain (39). If symptomatic, management involves division and reanastomosis of the subclavian artery to the carotid artery.

\section{Left circumflex aorta}

In normal circumstances, the dorsal aorta persists on the side of aortic arch. However, left circumflex aorta is a rare variation which consists of left aortic arch and the right sided descending aorta. In this anomaly the aortic arch itself courses behind esophagus. The vascular ring is completed by a segment of aortic arch on the left side along with a retroesophageal segment of aortic arch, ductus arteriosus on the right, and pulmonary artery on the anterior aspect (40). The embryological basis of this pathology is regression of the right fourth branchial arch between right common carotid and right subclavian artery along with persistence of the right ductus arteriosus and right dorsal aorta. However, if there is a left sided ductus, the vascular ring remains incomplete. The left aortic arch with circumflex aorta may result in severe tracheobronchial compression leading to respiratory compromise, especially in the pediatric patients, necessitating surgery (41). In such patients, compression may be relieved by division of the ductus or ligamentum arteriosum.

\section{Right aortic arch (RAA)}

Right sided aortic arch is a rare aortic anomaly with a reported incidence of $0.1 \%$ (42). There are three main subtypes based on Edward's model: type I-RAA with mirror image branching; type II-RAA with aberrant left subclavian artery; and type III-RAA with isolated subclavian artery. Type II is the most common subtype (43). Other rare subtypes include RAA with isolation of innominate artery and right circumflex aorta.

\section{Type I-RAA with mirror image branching}

RAA with mirror image branching is the result of regression of the left fourth branchial arch between left ductus and dorsal aorta. The branching pattern is exactly opposite of the left aortic arch with normal branching pattern.

Here the first branch is the left brachiocephalic artery followed by the right common carotid and finally the right subclavian artery (Figure 7). Usually there is a left ductus arising from left brachiocephalic artery and coursing anteriorly with no evidence of tracheo-esophageal compression (17). Sometimes, however, the ductus can be right sided, and in extremely rare situations, bilateral ductii may also be seen. Contrary to the popular belief that this anomaly does not result in the formation of a complete vascular ring, it may form a complete vascular ring in rare scenarios, e.g., if the left ductus arises from the descending thoracic aorta and courses posterior to the esophagus to join the left pulmonary artery (44).

Almost always, this malformation is associated with intracardiac defects like truncus arteriosus, tetralogy of Fallot with or without pulmonary stenosis, tricuspid atresia and it often is related to certain chromosomal anomalies like 22q11 deletion syndromes $(17,45)$. Isolated cases of RAA with mirror image branching do not require any treatment.

\section{Type II-RAA with aberrant left subclavian artery}

This is the most common subtype of RAA (46). The defect is due to the abnormal involution of left fourth arch in between the left subclavian and left common carotid artery. In this the first branch to arise is right common carotid, followed by right subclavian artery, left common carotid, and the last branch is the left subclavian artery that takes a retroesophageal course to reach on the left side (47). The ductus is usually on the right side, and with no formation of vascular ring. This subtype, unlike type I variation, is rarely associated with other cardiac malformations (48). 

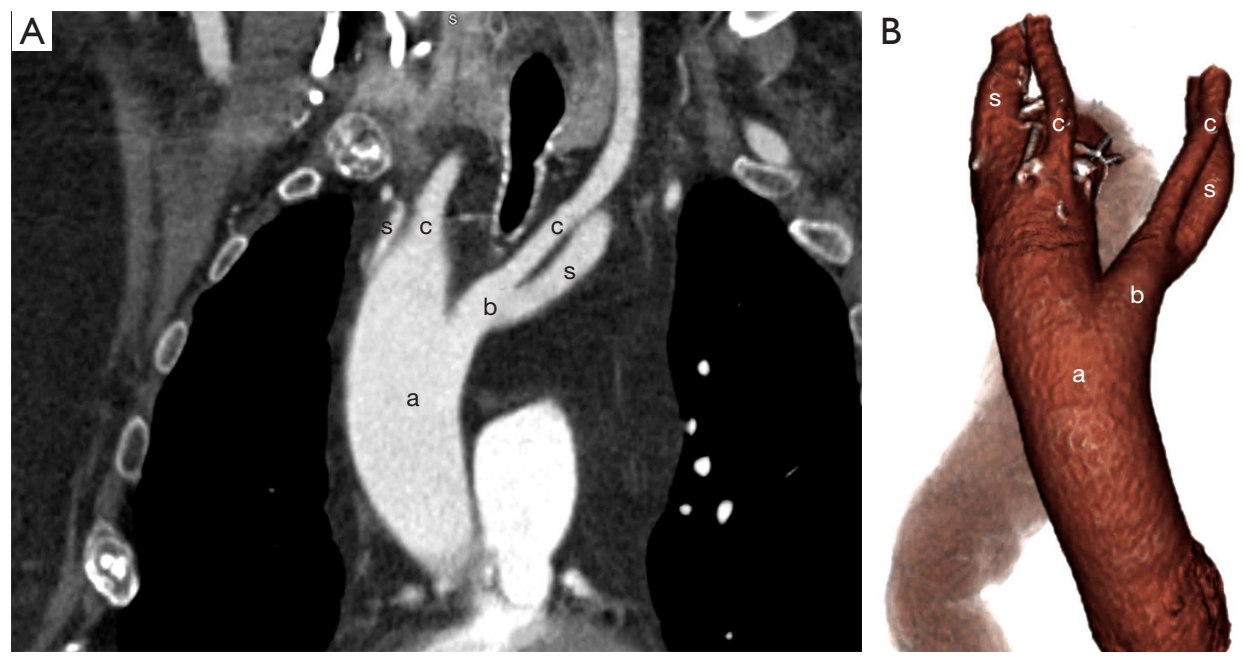

Figure 7 Right aortic arch with mirror branching: a 45-year-old female with acute onset chest pain, CTA to rule out dissection. Oblique coronal CTA (A) and 3D (B) images show a right sided aortic arch (a) with mirror branching. Note left brachiocephalic trunk (b), bilateral common carotid arteries (c) and subclavian arteries (s).
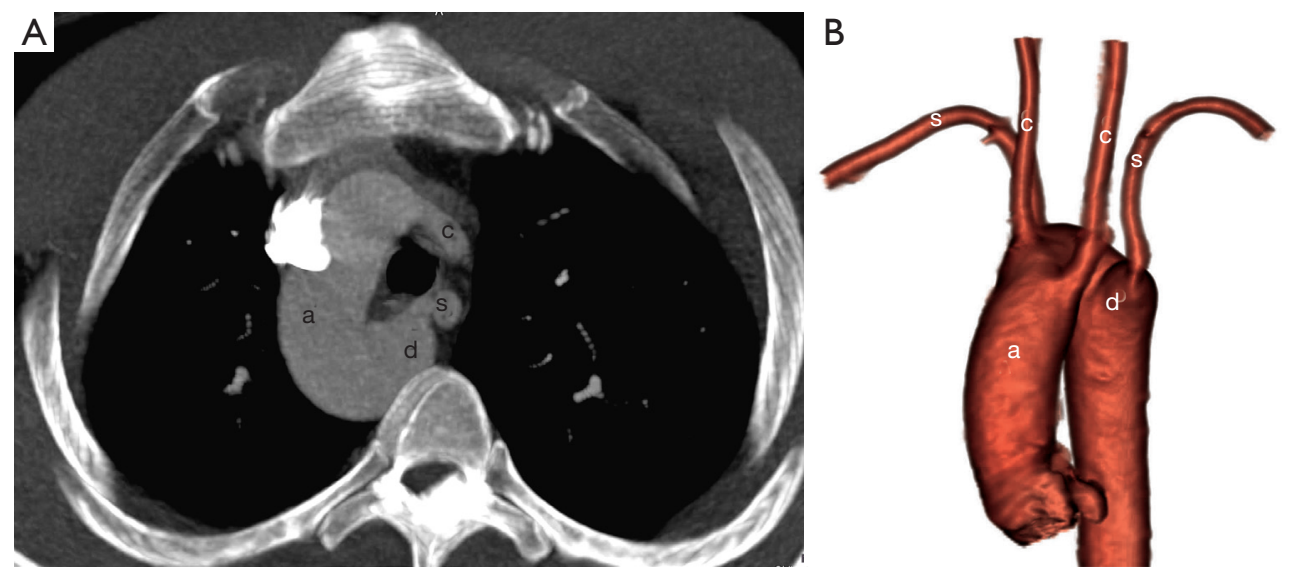

Figure 8 Right aortic arch with aberrant origin of left subclavian artery: a 38-year-old female with chest CT performed for dysphagia. Axial CT (A) shows right sided aortic arch (a) and aberrant origin of left subclavian artery (s) with dilatation at its origin called diverticulum of Kommerell (d). Note left common carotid artery (c). 3D MRA (B) shows right sided aortic arch (a) with diverticulum of Kommerell (d) at the origin of left subclavian artery. Note bilateral common carotid arteries (c) and subclavian arteries (s).

\section{$R A A$ with aberrant subclavian artery arising from diverticulum of Kommerell}

This is a classic malformation originally mentioned by Kommerell in which the aberrant left subclavian artery arises from retroesophageal diverticulum and runs a retroesophageal course (Figure 8). The complete vascular ring is formed by the segment of the ascending aorta anteriorly, Kommerell diverticulum posteriorly and the ligamentum arteriosum coursing on the left side. This results in complete encircling of trachea and esophagus $(49,50)$. If the diverticulum is aneurysmal, it may itself cause compression of esophagus or else it may rupture spontaneously (51). Thus, it is pertinent to report the presence or absence of retroesophageal diverticulum as it may have prognostic implications. In symptomatic patients, the diverticulum is resected and it is followed by aortopexy of the aortic arch. Alternatively, after dividing the diverticulum, the left subclavian artery can be reanastomosed to the left carotid artery. 


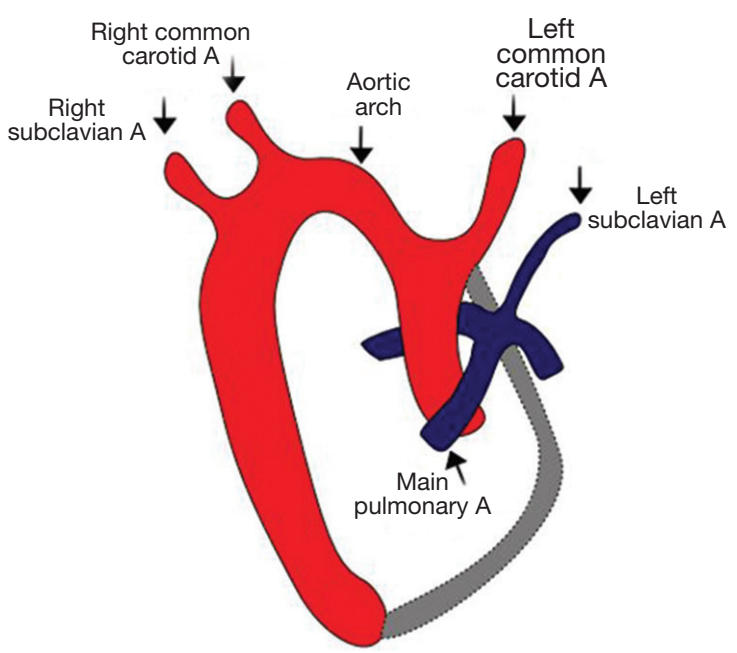

Figure 9 Schematic representation of right aortic arch with isolated left subclavian artery. This malformation is the result of involution of the left aortic arch at two levels, both between the left common carotid and left subclavian arteries, and distal to the left ductus arteriosus and left subclavian artery. The left subclavian artery arises from the pulmonary artery via a ductus arteriosus.

\section{Type III-RAA with isolated subclavian artery}

RAA with isolated subclavian artery is the result of regression of embryological left arch at two segments on either side of left subclavian artery (Figure 9). The left subclavian artery arises either from the vertebral artery or from the ductus arteriosus (52). The patients have features of subclavian steal syndrome and complain of weakness in the left arm $(39,53)$. Phase contrast magnetic resonance angiography if performed will reveal flow reversal in the left vertebral artery. Unlike type II RAA, type III is associated with increased incidence of congenital heart diseases like truncus arteriosus, tricuspid atresia or tetralogy of Fallot (54). The treatment strategy involves creation of a bypass graft to connect the common carotid and subclavian artery.

\section{Right circumflex aorta}

RAA can be associated with a contralateral left sided descending thoracic aorta. Right circumflex aorta is more common as compared to left circumflex aorta (55). To reiterate, in this type of malformation, it is not the subclavian artery but the aortic arch that takes a retroesophageal course. After crossing, it gives rise to a left sided ductus that usually arises from Kommerell diverticulum opposite to the side of aortic arch and completes the vascular ring (56). The subclavian artery may be normally positioned or may have an aberrant origin (Figure 10). The embryological theory behind this is the persistence of the left sided dorsal aorta leading to descending thoracic aorta coursing on the left side (57). The closest differential is double aortic arch with an atretic left arch segment. In such cases it is pertinent to inform the surgeon about the probability of an atretic left arch, especially when the preoperative anatomy is not conclusive (57). The treatment planning is similar to that of the left circumflex aorta which involves division of the ligament and ductus arteriosus to relieve the compression.

\section{Double aortic arch}

Double aortic arch is easily explainable by Edward's theoretical model in which both the right and the left fourth branchial arches persist leading to the so called double aortic arch, each one to be seen on either side of trachea (17). The common carotid and subclavian artery arises from the ipsilateral arches on both sides (Figure 11). This arrangement encircles the trachea and esophagus completely and is the most common cause of complete vascular ring (58). The ductus is usually on the left side and is seen ipsilateral to the side of atretic arch (43). Similarly, the descending thoracic aorta is contralateral to the side of dominant arch. The two aortic arches can be of the same caliber, or else one of the arches may be atretic or hypoplastic. Most commonly, the right arch is dominant and is more cranial as compared to the left arch (59). Clinically, the infants present early in life with barky cough and respiratory stridor.

\section{Double aortic arch with partial/complete atresia of one arch}

Double aortic arch may have atresia of one of the arches, more commonly of the left that persist as fibrous or ligamentous remnant (60). Contrast enhanced CT or MR angiography is able only to depict the patent vessels and is not capable in assessing the ligamentous atretic segments directly. Thus, it is imperative to look for secondary signs that will help in detecting the atretic residual remnant and also in differentiating it from close mimics.

\section{Atretic double aortic arch and $R A A$ with mirror image branching}

If the atretic segment involves the left arch and is distal to origin of the left subclavian artery, it may be extremely 

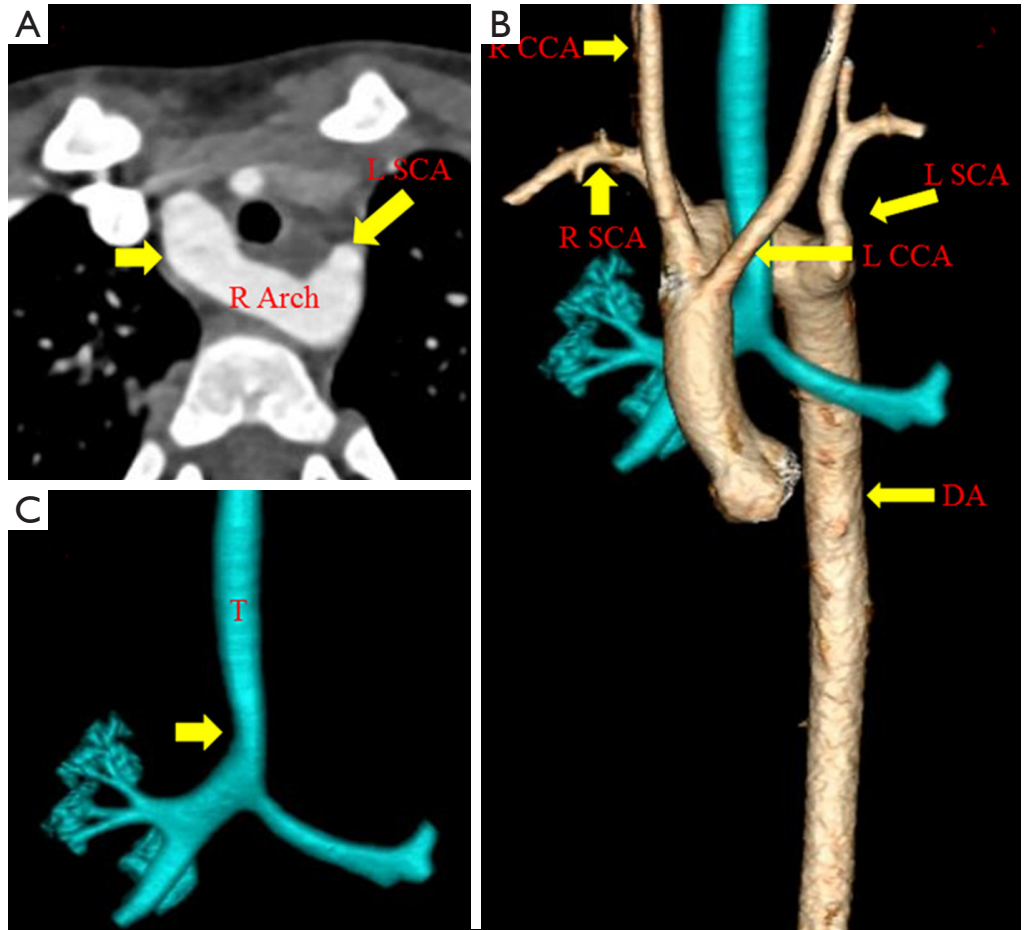

Figure 10 Right circumflex aorta, a 2-year-old male child with history of chronic cough. CTA (A) demonstrates right aortic arch (short arrow) crossing over to the left side posterior to trachea. After crossing over, the arch gives rise to aberrant left subclavian artery. Volumetric 3D CTA (B) shows right aortic arch with descending aorta on the left side with evidence of tracheal compression by the crossing aortic arch (C). L SCA, left subclavian artery; R arch, right aortic arch; R SCA, right subclavian artery; R CCA, right common carotid artery; L CCA, left common carotid artery; DA, descending aorta; T, trachea.
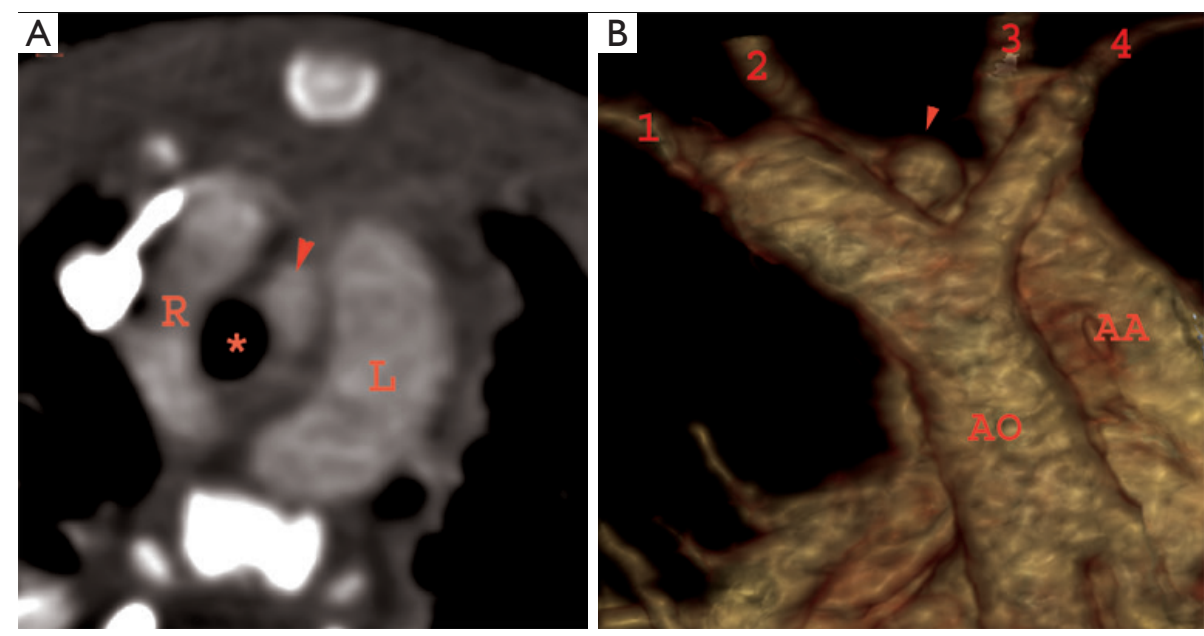

Figure 11 Double aortic arch: a 15-month-old female with failure to thrive and abnormal mediastinal contour on chest radiograph. Axial CTA image (A) showing double aortic arch encircling the airway $\left(^{*}\right)$ with left arch $(\mathrm{L})$ being dominant. Volume rendered image (B) showing four vessel sign in the same patient. Note is made of patent ductus arteriosus (PDA) from left arch (arrowhead). 1, left subclavian artery; 2, left common carotid artery; 3, right common carotid artery; 4, right subclavian artery; R, right; L, left; AO, descending thoracic aorta; AA, ascending aorta. 

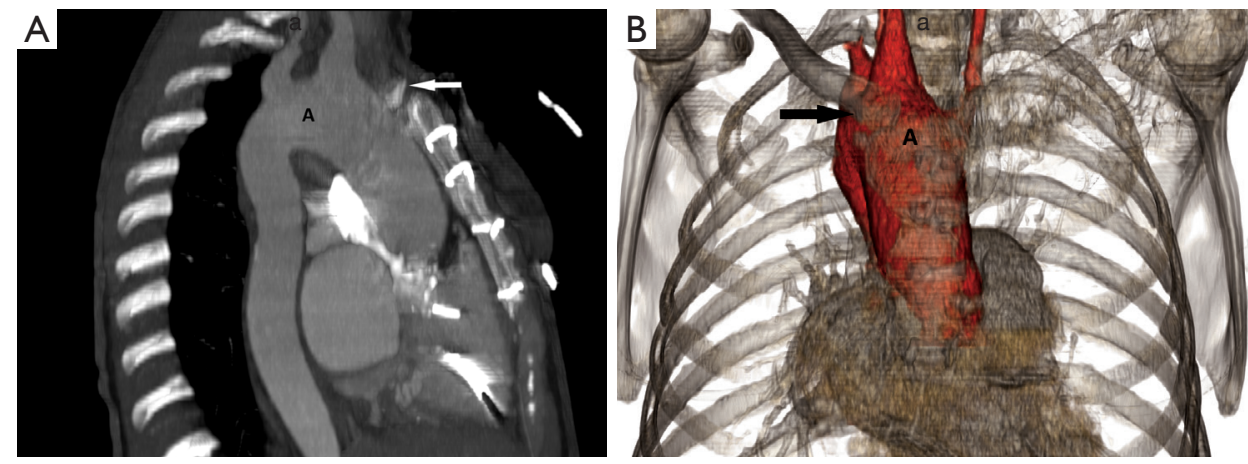

Figure 12 Cervical aortic arch: a 51-year-old male with known ascending aortic aneurysm on follow up. Oblique sagittal MIP CTA (A) and coronal 3D (B) images show high location of a right sided aortic arch (A) extending just above the medial end of right clavicle (arrow).

difficult to differentiate it from the RAA with mirror image branching. In these scenarios we may look for ancillary findings that can help us in distinguishing between the two.

(I) Four vessel sign: It is an important diagnostic clue in the diagnosis of complete vascular ring. The assessment is made on the basis of axial images taken just above the level of aortic arch. It consists of two vessels visible on each side. This symmetric pattern is predictive of double aortic arch. However, in patients with RAA with mirror image branching, there is only one vessel on the left side and that is a left brachiocephalic artery $(61,62)$.

(II) Ductal diverticulum: in double aortic arch with left arch atresia, the ductal diverticulum is seen projecting superiorly while in RAA with mirror image branching, it is projecting anteriorly towards the pulmonary artery (63).

(III) Course: atretic left arch is placed more posteriorly while the left brachiocephalic artery is positioned more anteriorly in patients with RAA with mirror image branching (64).

(IV) Subclavian artery sign: the course of subclavian artery is distorted in patients with double aortic arch as there it takes an inferior course (representing the embryological persistent segment of left atretic arch) and then turns towards cephalad. There may be a tenting of the artery seen projecting inferiorly and that may represent persistent connection to the atretic $\operatorname{arch}(65)$.

\section{Atretic double aortic arch and $R A A$ with aberrant subclavian artery}

If the atresia is between the left common carotid and left subclavian artery, it may be difficult to differentiate this entity from the aberrant left subclavian artery in RAA $(57,64)$. In such cases, it is necessary to provide the information to the operating surgeon about any such possibility, so that during surgery, he may cautiously look for the atretic segment that needs to be resected. The treatment usually involves resection of the lesser arch and division of the ligamentum arteriosum via lateral thoracotomy approach.

\section{Cervical aortic arch}

In cervical aortic arch, the aortic arch is seen at an abnormally high position reaching at or just above the level of clavicles (Figure 12). It is presumed to be arising either from persistence of the second or third branchial arches with regression of the normal fourth arch, or from failure of the caudal migration of the normally developing fourth arch (66). The cervical aortic arch complex consists of high position of aortic arch in the superior mediastinum, separate origin of contralateral carotid arteries, presence of aberrant contralateral subclavian artery from the aortic diverticulum and retroesophageal descending aorta coursing contralateral to the side of the aortic arch $(67,68)$. The clinical presentation is of a pulsatile neck mass. Majority of patients do not require surgery as the anomaly does not result in compressive symptoms. The cervical aortic arch is commonly seen with RAA and is usually not associated with other intracardiac defects. However, it may be associated with 22q11 microdeletion syndromes (69).

\section{IAA}

The presence of complete aortic luminal discontinuity 
between ascending and descending aorta is termed as interruption of the aortic arch. However, there may be sometimes a remnant fibrous cord. The source of blood flow to the descending thoracic aorta is mostly via the PDA. It is classified into three types on the basis of the level of aortic interruption. The radiological report should include description about the level of interruption, distance between

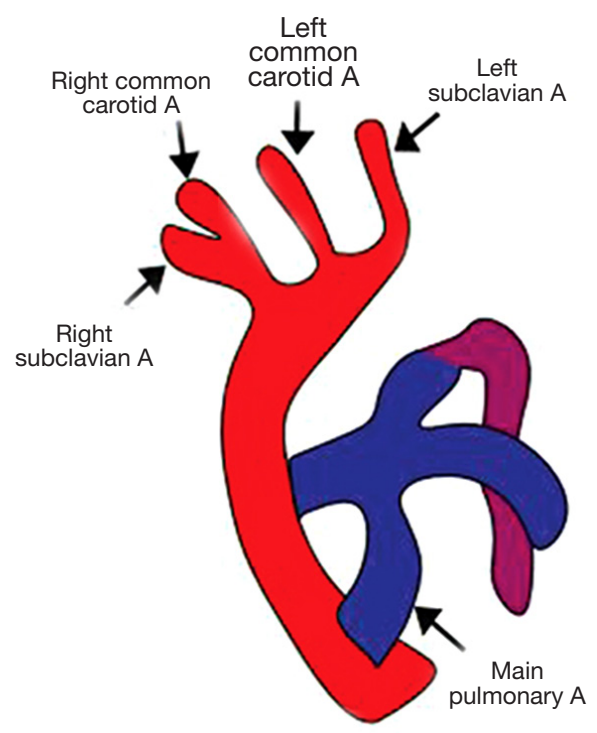

Figure 13 Schematic representation of the type A interrupted aortic arch. Type A arch involves interruption distal to the left subclavian artery. A large PDA perfuses the descending aorta. aortic arch and descending thoracic aorta, branching pattern and other associated anomalies, if any. The treatment involves surgical repair using a bypass graft, stent or end-toend anastomosis.

Type A interruption: it is characterized by the level of interruption distal to the left subclavian artery (Figures 13,14).

Type B interruption: the level of discontinuity is between left common carotid and left subclavian artery. (Figures 15,16).

Type $\mathrm{C}$ interruption: it involves luminal interruption between right brachiocephalic and left common carotid artery (Figure 17).

Type $B$ is the most common subtype in the infants and type $\mathrm{A}$ in the adult patients (70). IAA may be associated with other congenital heart diseases like ventricular septal defect, PDA and bicuspid aortic valve. Type B is also associated with subaortic stenosis due to malalignment of outlet conal septum (71). IAA is generally seen in patients with $22 \mathrm{q} 11$ deletion syndrome, predominantly involving type B (69).

The entity should be diagnosed in multiple planes since the presence of a large PDA may look like an arch, and that may lead to an erroneous diagnosis. The PDA is more flat in appearance as compared to the normal arch (72).

\section{IAA and atresia of aortic arch}

IAA presents itself in the form of complete discontinuity and true absence of any communication between transverse and
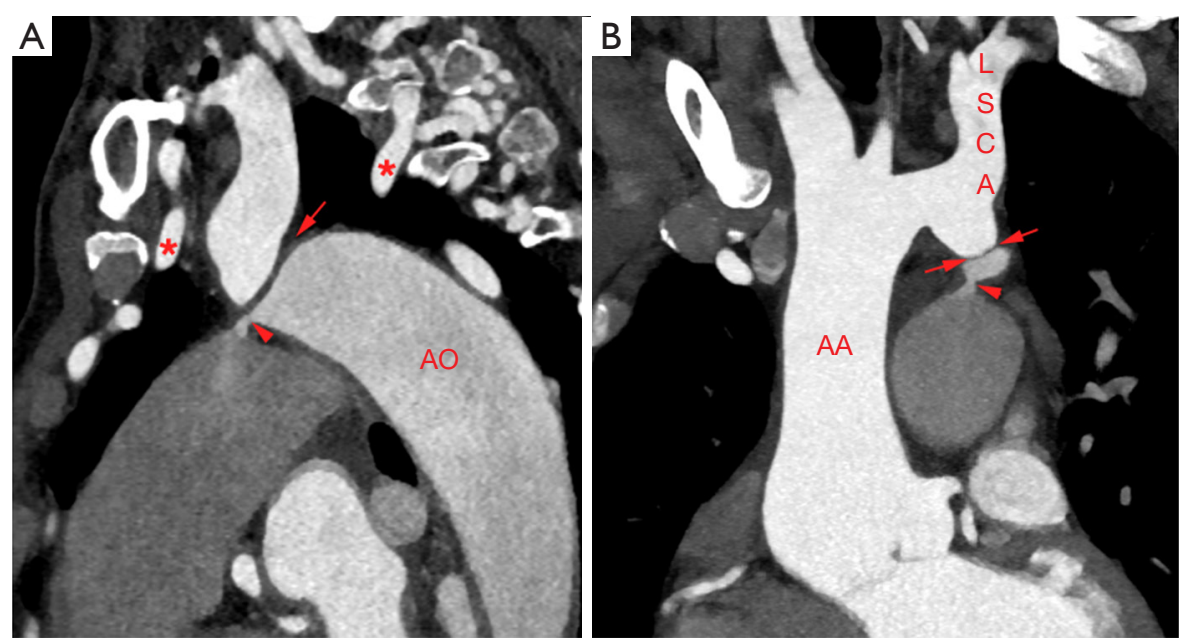

Figure 14 Type A interrupted aortic arch: sagittal oblique (A) and coronal (B) CTA images showing type A aortic interruption (arrows) with reformation of descending thoracic aorta (AO) through thin PDA (arrowhead) and presence of collaterals $\left(^{*}\right)$. LSCA, left subclavian artery; AA, ascending aorta. 
descending thoracic aorta. In case of arch atresia, however, there is evidence of discontinuity between the aortic arch and descending aorta in the form of persistent anatomic fibrous remnant. This fibrous remnant is not visualized

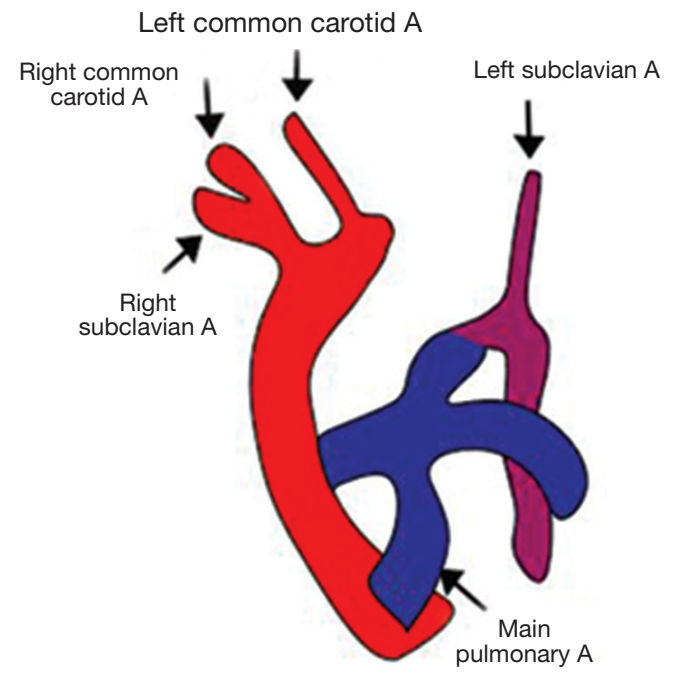

Figure 15 Schematic representation of the type B interrupted aortic arch. Type B arch involves interruption between left common carotid and left subclavian artery. Descending aorta is perfused by a large patent ductus arteriosus (PDA). Left subclavian artery arises from the descending aorta. on imaging as there is no patent lumen. Nevertheless, the presence of diverticulum on both sides of the distant aortic segments may point towards presence of arch atresia (73).

\section{Hypoplastic aortic arch}

Aortic arch hypoplasia is defined in terms of relativity of the external diameter of ascending aorta, assuming ascending aorta is of the normal caliber. For the proximal arch hypoplasia, the external diameter is $<60 \%$, for distal arch $<50 \%$ and for isthmic segment it is $<40 \%$ of the external diameter of ascending aorta (Figure 18). There may be physiological tapering of distal segment of the distal arch in the first three months of life $(17,74)$.

\section{Coarctation and pseudocoarctation of aorta}

The coarctation of aorta is described as a tight stenosis just distal to the left subclavian artery (juxtaductal). This entity is hemodynamically significant if blood pressure gradient is more than $20 \mathrm{mmHg}$ between the upper and lower extremity (75) and it leads to development of the intercostal collateral vessels that serve to bypass the site of coarctation in order to maintain blood flow to the distal descending thoracic aorta (Figure 19). CTA is able to provide anatomical details of the site and degree of narrowing, relationship of the coarctation
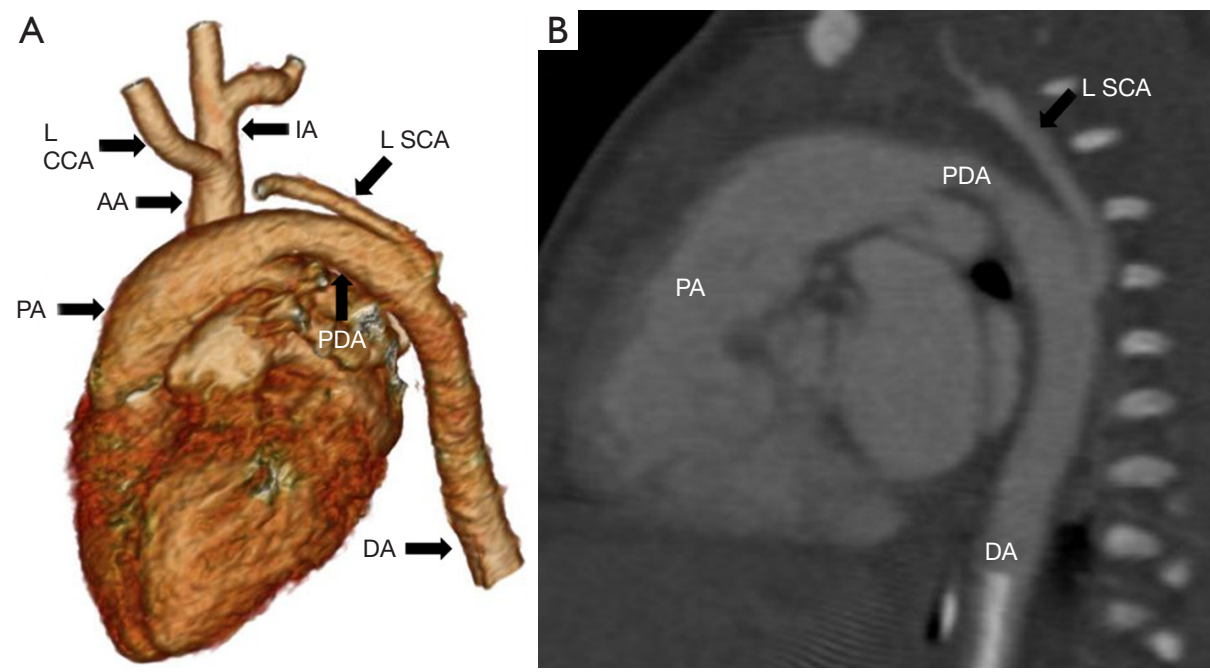

Figure 16 Type B interrupted aortic arch in a term female born at 39 weeks of gestation with DiGeorge's syndrome. Volumetric 3D CTA (A) depicts interruption in aortic arch distal to the origin of left common carotid artery. Descending aorta and left subclavian artery are perfused by a large PDA. Sagittal oblique reformatted CTA (B) shows perfusion of descending aorta from a large PDA. Left subclavian artery arises from the descending aorta. L SCA, left subclavian artery; PA, main pulmonary artery; IA, right innominate artery; L CCA, left common carotid artery; DA, descending aorta; AA, ascending aorta; PDA, patent ductus arteriosus. 
to the arch vessels, associated pseudoaneurysms, if any, and it provides information about the collateral vessels (76). For post-operative evaluation following stent placement, CT is the better choice as compared to MRA. Similarly, for functional evaluation, MRA is a better choice compared to CT as phase contrast MRA can assess the collateral flow by comparing the flow in the proximal abdominal aorta with the flow just proximal to the narrowed aortic segment (77). Coarctation of

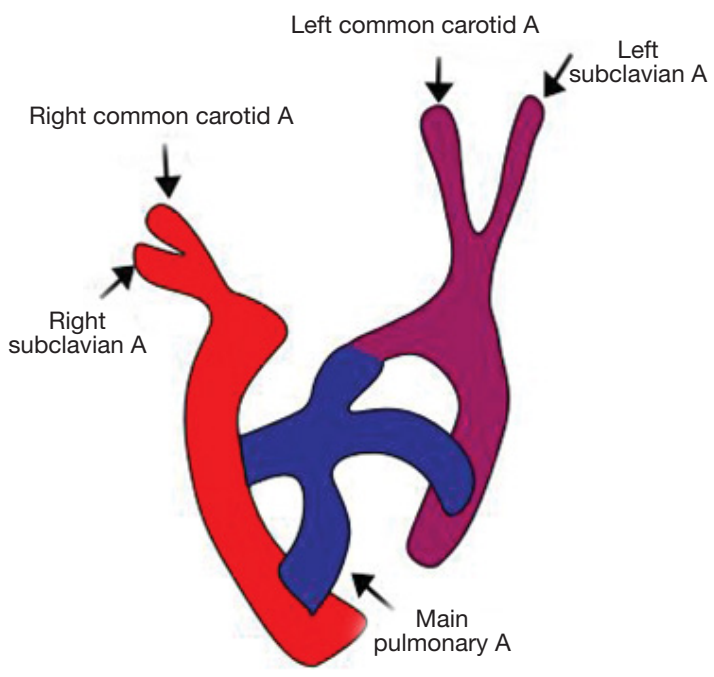

Figure 17 Schematic representation of the type C interrupted aortic arch. Type $\mathrm{C}$ arch involves interruption between right innominate and left common carotid artery. Left common carotid and left subclavian artery as well as descending aorta are all perfused via a large patent ductus arteriosus (PDA). aorta is commonly associated with bicuspid aortic valve, arch hypoplasia, ventricular and atrial septal defects, and mitral valve abnormalities (78).

In contrast pseudocoarctation is hemodynamically insignificant. It encompasses kinking and elongation of aortic arch with no evidence of collateral vessels. It differs from true coarctation since there is no evidence of left ventricular hypertrophy or ascending aortic aneurysm, absence of collateral vessels, and no pressure gradient across the kinked arch. Moreover, both CTA and MRA would be able to assess the elongated arch and differentiate it from the true coarctation of aorta $(79,80)$. Usually it is asymptomatic, but in rare circumstances, there may be aneurysm formation that may rupture necessitating surgical intervention (81). Elongation of transverse aortic arch with slightly distal origin of left subclavian artery may also be seen in patients with turner syndrome (82).

\section{Persistent fifth aortic arch (PFAA)}

PFAA is a rare anomaly first described in 1969 as a doublelumen aortic arch. It is located below the 4th arch which forms the right subclavian artery and the definitive aortic arch, and it is above the 6th arch whose derivatives are the left pulmonary artery or ductus (83).

\section{Clinical perspective}

The clinical presentation depends on whether the connection is a systemic to systemic or systemic to
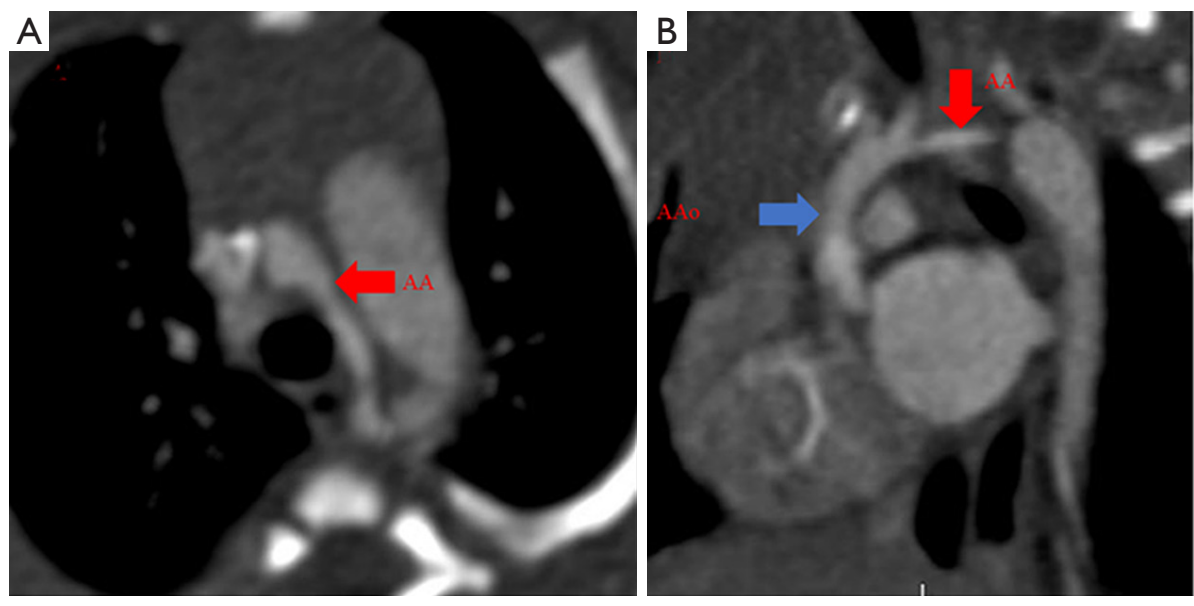

Figure 18 A 46-day-old female with hypoplastic aortic arch. CTA axial (A) and oblique reformatted multiplanar CTA (B) shows evidence of hypoplasia of both the aortic arch (red arrow) and ascending aorta (blue arrow). AA, aortic arch; AAo, ascending aorta. 


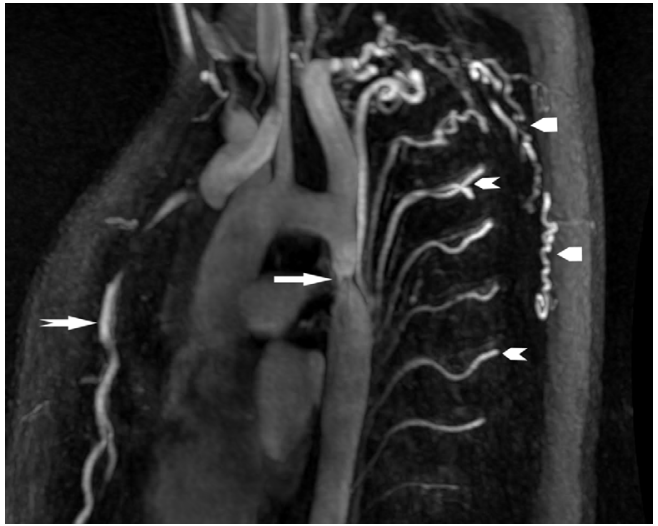

Figure 19 Coarctation of aorta: a 21-year-old female with severe hypertension. Sagittal MIP MRA shows focal stenosis in the proximal descending thoracic aorta (arrow). Note the prominent internal mammary artery (notched arrow), intercostal arteries (notched arrowheads) and other chest wall collaterals (arrowheads). pulmonary system. PFAA is hemodynamically beneficial when associated with coarctation of the aorta or with an IAA. The systemic-to-pulmonary arterial connection is beneficial when associated with pulmonary atresia or with tricuspid atresia (Figure 20). Patients with right ventricular outflow obstruction develop multiple aorto-pulmonary collaterals as a way to maintain pulmonary circulation (84).

PFAA can be either right-sided or left-sided. There are three types of PFAA (85): (I) double-lumen aortic arch with both lamina patent; (II) atresia and interruption of the superior arch with patent inferior (persistent fifth) arch; (III) systemic-to-pulmonary arterial connection arising proximal to the first brachiocephalic artery. Type I PFAA is the most common form. Type III has often been described in association with pulmonary atresia and ventricular septal defect. PFAA may be associated with pulmonary atresia,
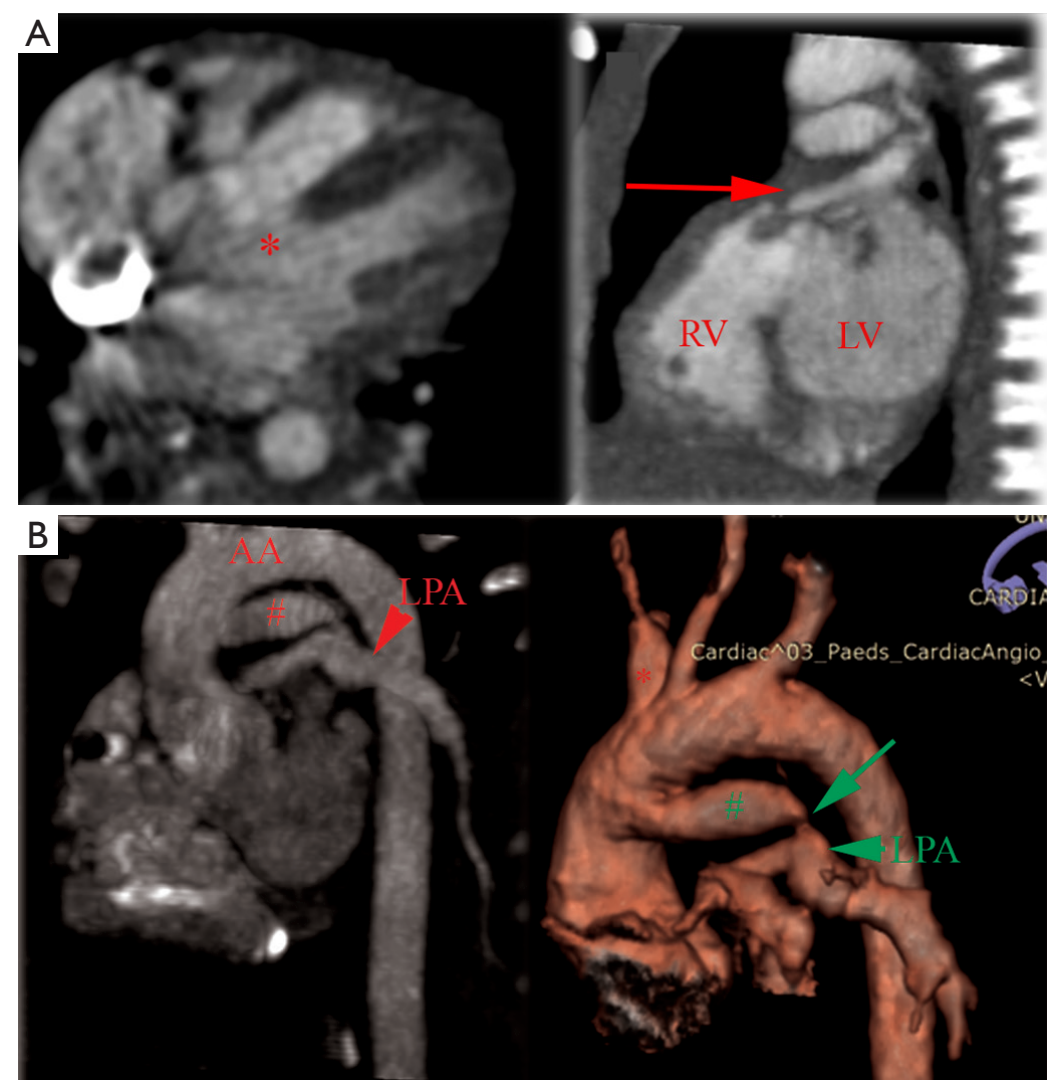

Figure 20 Persistent fifth aortic arch in a patient with pulmonary atresia and ventricular septal defect: a 2-month-old male with antenatally diagnosed cardiac malformation, referred for further management. CTA images (A) show subaortic ventricular septal defect $\left(^{*}\right)$ and pulmonary atresia (red arrow). CTA and 3D (B) images show vascular channel (\#) extending from the undersurface of aortic arch (AA) opposite to brachiocephalic artery origin $\left(^{*}\right)$ to the left pulmonary artery (LPA) with tight stenosis at insertion (green arrow). RV, right ventricle; $\mathrm{LV}$, left ventricle 
tricuspid atresia, coarctation of aorta, IAA, ventricular septal defect, PDA, atrioventricular septal defect, tetralogy of Fallot and persistent truncus arteriosus (86).

\section{Imaging characteristics of PFAA}

For a true fifth arch, its origin must be proximal to the brachiocephalic artery. It takes a tortuous course, always lies in extra pericardial sac, and terminates in either the dorsal aorta or the pulmonary artery via the ductus arteriosus (87).

The differential diagnosis includes aortopulmonary window (AP Window) and PDA. In PFAA, communicating artery connects distally to the origin of the pulmonary arteries. In the case of an $\mathrm{AP}$ window, the connection is along the pulmonary trunk and bifurcation of the pulmonary arteries.

A high AP window, however, may extend to the origin of the right pulmonary artery. A PDA would communicate distally to the arch arteries while the communicating artery in PFAA connects the aorta proximally to the origin of the head and neck vessels.

PFAA may result in the formation of a complete vascular ring and thus closely mimic the double aortic arch. This occurs due to persistence of a large posterior aortic diverticulum that completes the fifth arch posteriorly forming a ring. However, in double aortic arch both arches give rise to ipsilateral carotid and subclavian arteries; where as in patients with PFAA forming a complete ring the left subclavian artery is arising from the fifth aortic arch. The left common carotid, right common carotid and the right subclavian arteries arise from the right arch. This type of PFAA has been classified as type A-4 (88).

\section{Conclusions}

Congenital aortic arch anomalies comprise a rare spectrum of cardiovascular diseases. Imaging is therefore required to elucidate the complex aortic arch anomalies, abnormal branching pattern, their relationship with trachea and esophagus, and other associated cardiac and non-cardiac pathologies. Both CT and MR angiography provide a detailed assessment of the high risk vascular ring features like the double aortic arch, retroesophageal Kommerell diverticulum and circumflex aorta. By making use of three dimensional capabilities of both CTA and MRA, anatomical relationship of the aortic arch with trachea and esophagus can be defined properly thus avoiding conventional techniques like esophagogram and, possibly, bronchoscopy. MRA avoids radiation therefore is more suitable, especially for the pediatric patients. It also suits long term serial follow-up. However, longer acquisition times, potential need for anesthesia in infants, small children, and claustrophobia are some of the drawbacks of MRA. CT angiography is therefore ideal in these situations and provides excellent spatial resolution. Modern CT scanners with state-of-the-art protocols result in reduction of the radiation exposure which may make utilization more attractive in this population. The radiology report should describe all the anatomical and functional details, high risk vascular ring characteristics, and differential considerations in order to best aid preoperative surgical planning.

\section{Acknowledgements}

We would like to acknowledge Dr. Shawn Sato, Department of Pediatric Radiology, University of Iowa Hospitals \& Clinics for contributing image number 10 and 16.

\section{Footnote}

Conflicts of Interest: The authors have no conflicts of interest to declare.

\section{References}

1. Lim HK, Ha HI, Hwang HJ, et al. Feasibility of highpitch dual-source low-dose chest CT: Reduction of radiation and cardiac artifacts. Diagn Interv Imaging 2016;97:443-9.

2. McLaren CA, Elliott MJ, Roebuck DJ. Vascular compression of the airway in children. Paediatr Respir Rev 2008;9:85-94.

3. Fogel MA, Pawlowski TW, Harris MA, et al. Comparison and Usefulness of Cardiac Magnetic Resonance Versus Computed Tomography in Infants Six Months of Age or Younger With Aortic Arch Anomalies Without Deep Sedation or Anesthesia. Am J Cardiol 2011;108:120-5.

4. Leonardi B, Secinaro A, Cutrera R, et al. Imaging modalities in children with vascular ring and pulmonary artery sling. Pediatr Pulmonol 2015;50:781-8.

5. Zucker EJ, Cheng JY, Haldipur A, et al. Free-breathing pediatric chest MRI: Performance of self-navigated goldenangle ordered conical ultrashort echo time acquisition. J Magn Reson Imaging 2018;47:200-9.

6. Cheng JY, Hanneman K, Zhang T, et al. Comprehensive motion-compensated highly accelerated 4D flow MRI with ferumoxytol enhancement for pediatric congenital heart disease. J Magn Reson Imaging 2016;43:1355-68. 
7. Hahn AD, Higano NS, Walkup LL, et al. Pulmonary MRI of neonates in the intensive care unit using 3D ultrashort echo time and a small footprint MRI system. J Magn Reson Imaging 2017;45:463-71.

8. Krishnamurthy R, Bahouth SM, Muthupillai R. 4D Contrast-enhanced MR Angiography with the Keyhole Technique in Children: Technique and Clinical Applications. Radiographics 2016;36:523-37

9. Olchowy C, Cebulski K, Łasecki M, et al. The presence of the gadolinium-based contrast agent depositions in the brain and symptoms of gadolinium neurotoxicity A systematic review. Mohapatra S, editor. PLoS One 2017;12:e0171704.

10. Gulani V, Calamante F, Shellock FG, et al. Gadolinium deposition in the brain: summary of evidence and recommendations. Lancet Neurol 2017;16:564-70.

11. Bultman EM, Klaers J, Johnson KM, et al. Non-contrast enhanced 3D SSFP MRA of the renal allograft vasculature: a comparison between radial linear combination and Cartesian inflow-weighted acquisitions. Magn Reson Imaging 2014;32:190-5.

12. Tandon A, Hashemi S, Parks WJ, et al. Improved highresolution pediatric vascular cardiovascular magnetic resonance with gadofosveset-enhanced 3D respiratory navigated, inversion recovery prepared gradient echo readout imaging compared to $3 \mathrm{D}$ balanced steady-state free precession readout imaging. J Cardiovasc Magn Reson 2016;18:74.

13. Pasqua AD, Barcudi S, Leonardi B, et al. Comparison of contrast and noncontrast magnetic resonance angiography for quantitative analysis of thoracic arteries in young patients with congenital heart defects. Ann Pediatr Cardiol 2011;4:36-40.

14. Kawel N, Jhooti P, Dashti D, et al. MR-imaging of the thoracic aorta: 3D-ECG- and respiratory-gated bSSFP imaging using the CLAWS algorithm versus contrastenhanced 3D-MRA. Eur J Radiol 2012;81:239-43.

15. Kau T, Sinzig M, Gasser J, et al. Aortic Development and Anomalies. Semin Intervent Radiol 2007;24:141-52.

16. Jakanani GC, Adair W. Frequency of variations in aortic arch anatomy depicted on multidetector CT. Clin Radiol 2010;65:481-7.

17. Hanneman K, Newman B, Chan F. Congenital Variants and Anomalies of the Aortic Arch. RadioGraphics 2017;37:32-51.

18. Türkvatan A, Büyükbayraktar FG, Ölçer T, et al. Congenital Anomalies of the Aortic Arch: Evaluation with the Use of Multidetector Computed Tomography. Korean
J Radiol 2009;10:176.

19. Bravo C, Gámez F, Pérez R, et al. Fetal Aortic Arch Anomalies. J Ultrasound Med 2016;35:237-51.

20. Kellenberger CJ. Aortic arch malformations. Pediatr Radiol 2010;40:876-84.

21. Ming Z, Yumin Z, Yuhua L, et al. Diagnosis of congenital obstructive aortic arch anomalies in Chinese children by contrast-enhanced magnetic resonance angiography. J Cardiovasc Magn Reson 2006;8:747-53.

22. Predey TA, McDonald V, Demos TC, et al. CT of congenital anomalies of the aortic arch. Semin Roentgenol 1989;24:96-113.

23. Dominguez R, Oh KS, Dorst JP, et al. Left aortic arch with right descending aorta. AJR Am J Roentgenol 1978;130:917-20.

24. Natsis KI, Tsitouridis IA, Didagelos MV, et al. Anatomical variations in the branches of the human aortic arch in 633 angiographies: clinical significance and literature review. Surg Radiol Anat 2009;31:319-23.

25. Moise MA, Hsu V, Braslow B, et al. Innominate artery transection in the setting of a bovine arch. J Thorac Cardiovasc Surg 2004;128:632-4.

26. Malone CD, Urbania TH, Crook SE, et al. Bovine aortic arch: A novel association with thoracic aortic dilation. Clin Radiol 2012;67:28-31.

27. Hornick M, Moomiaie R, Mojibian H, et al. 'Bovine' Aortic Arch - A Marker for Thoracic Aortic Disease. Cardiology 2012;123:116-24.

28. Nagpal P, Khandelwal A, Saboo SS, et al. Modern imaging techniques: applications in the management of acute aortic pathologies. Postgrad Med J 2015;91:449-62.

29. Yuan SM. Aberrant Origin of Vertebral Artery and Its Clinical Implications. Braz J Cardiovasc Surg 2016;31:52-9.

30. Tong E, Rizvi T, Hagspiel KD. Complex aortic arch anomaly: Right aortic arch with aberrant left subclavian artery, fenestrated proximal right and duplicated proximal left vertebral arteries-CT angiography findings and review of the literature. Neuroradiol J 2015;28:396-403.

31. Natsis K, Didagelos M, Gkiouliava A, et al. The aberrant right subclavian artery: cadaveric study and literature review. Surg Radiol Anat 2017;39:559-65.

32. Abraham V, Mathew A, Cherian V, et al. Aberrant subclavian artery: Anatomical curiosity or clinical entity. Int J Surg 2009;7:106-9.

33. Saeed G, Ganster G, Friedel N. Arteria lusoria aneurysm with truncus bicaroticus: surgical resection without restoring blood supply to the right arm. Tex Heart Inst J 2010;37:602-7. 
34. Rogers AD, Nel M, Eloff EP, et al. Dysphagia lusoria: a case of an aberrant right subclavian artery and a bicarotid trunk. ISRN Surg 2011;2011:819295.

35. Grathwohl KW, Afifi AY, Dillard TA, et al. Vascular rings of the thoracic aorta in adults. Am Surg 1999;65:1077-83.

36. Pati PK, Verghese MJ, George PV. Isolation of left subclavian artery with reversal of neurological and hemodynamic abnormalities after percutaneous closure. Indian Heart J 2014;66:477-80.

37. Saktheeswaran MK, Krishnamoorthy KM, Sivasankaran S. Isolation of the left subclavian artery: a rare aortic arch anomaly. Cardiol Young 2015;25:1203-5.

38. Bech AP, Op Den Akker J, Matthijsse PR. Isolation of the left subclavian artery from the pulmonary artery in a patient with CHARGE association. Congenit Anom (Kyoto) 2010;50:200-2.

39. Jesudian V, Ravikumar R, Kumar RS. Isolation of the left subclavian artery-origin from the left pulmonary artery by way of ductus arteriosus: multidetector row computed tomographic angiographic imaging. Pediatr Cardiol 2009;30:549-50.

40. McLeary MS, Frye LL, Young LW. Magnetic resonance imaging of a left circumflex aortic arch and aberrant right subclavian artery: the other vascular ring. Pediatr Radiol 1998;28:263-5.

41. Baird CW, Prabhu S, Buchmiller TL, et al. Direct Tracheobronchopexy and Posterior Descending Aortopexy for Severe Left Mainstem Bronchomalacia Associated With Congenital Pulmonary Airway Malformation and Left Circumflex Aortic Arch. Ann Thorac Surg 2016;102:e1-4.

42. D'Antonio F, Khalil A, Zidere V, et al. Fetuses with right aortic arch: a multicenter cohort study and meta-analysis. Ultrasound Obstet Gynecol 2016;47:423-32.

43. García-Guereta L, García-Cerro E, Bret-Zurita M. Multidetector Computed Tomography for Congenital Anomalies of the Aortic Arch: Vascular Rings. Rev Esp Cardiol Engl Ed 2016;69:681-93.

44. McElhinney DB, Hoydu AK, Gaynor JW, et al. Patterns of right aortic arch and mirror-image branching of the brachiocephalic vessels without associated anomalies. Pediatr Cardiol 2001;22:285-91.

45. Knight L, Edwards JE. Right aortic arch. Types and associated cardiac anomalies. Circulation 1974;50:1047-51.

46. Tyczyński P, Michałowska I, Wolny R, et al. Left aberrant subclavian artery. Systematic study in adult patients. Int J Cardiol 2017;240:183-6.

47. Masuoka H, Miyauchi A, Higashiyama T, et al. Rightsided aortic arch and aberrant left subclavian artery with or without a left nonrecurrent inferior laryngeal nerve. Head Neck 2016;38:E2508-11.

48. Cinà CS, Althani H, Pasenau J, et al. Kommerell's diverticulum and right-sided aortic arch: a cohort study and review of the literature. J Vasc Surg 2004;39:131-9.

49. Zhao J, Liao Y, Gao S. Right aortic arch with retroesophageal left ligamentum arteriosum. Tex Heart Inst J 2006;33:218-21.

50. Silva AF, Dos Santos JA. Aortic arch anomaly in an adult patient: a case of right aortic arch with aberrant left subclavian artery and Kommerell's diverticulum. Radiol Bras 2016;49:274-5.

51. Faistauer Â, Torres FS, Faccin CS. Right aortic arch with aberrant left innominate artery arising from Kommerell's diverticulum. Radiol Bras 2016;49:264-6.

52. Sun AM, Alhabshan F, Branson H, et al. MRI diagnosis of isolated origin of the left subclavian artery from the left pulmonary artery. Pediatr Radiol 2005;35:1259-62.

53. Arnoult AC, Blaise S. Late discovery of a rare anomaly of the right aortic arch and an isolated left subclavian artery. J Vasc Surg 2016;64:1853-4.

54. Carano N, Piazza P, Agnetti A, et al. Congenital pulmonary steal phenomenon associated with tetralogy of Fallot, right aortic arch, and isolation of the left subclavian artery. Pediatr Cardiol 1997;18:57-60.

55. Smith BM, Lu JC, Dorfman AL, et al. Rings and Slings Revisited. Magn Reson Imaging Clin N Am 2015;23:127-35.

56. Philip S, Chen SY, Wu MH, et al. Retroesophageal aortic arch: diagnostic and therapeutic implications of a rare vascular ring. Int J Cardiol 2001;79:133-41.

57. Gould SW, Rigsby CK, Donnelly LF, et al. Useful signs for the assessment of vascular rings on cross-sectional imaging. Pediatr Radiol 2015;45:2004-16.

58. Thankavel PP, Brown PS, Lemler MS. Left-Dominant Double Aortic Arch in Critical Pulmonary Stenosis and Ventricular Septal Defect. Pediatr Cardiol 2012;33:1469-71.

59. Singh C, Gupta M, Sharma S. Compression of trachea due to double aortic arch: demonstration by multi-slice CT scan (MSCT). Heart Lung Circ 2006;15:332-3.

60. Baraldi R, Sala S, Bighi S, et al. Vascular ring due to double aortic arch: a rare cause of dysphagia. Eur J Radiol Extra 2004;52:21-4.

61. Dillman JR, Attili AK, Agarwal PP, et al. Common and uncommon vascular rings and slings: a multi-modality review. Pediatr Radiol 2011;41:1440-54.

62. Browne LP. What is the optimal imaging for vascular rings and slings? Pediatr Radiol 2009;39:S191-5.

63. Newman B. MR of right aortic arch. Pediatr Radiol 
1996;26:367-9.

64. Schlesinger AE, Krishnamurthy R, Sena LM, et al. Incomplete double aortic arch with atresia of the distal left arch: distinctive imaging appearance. AJR Am J Roentgenol 2005;184:1634-9.

65. Holmes KW, Bluemke DA, Vricella LA, et al. Magnetic resonance imaging of a distorted left subclavian artery course: an important clue to an unusual type of double aortic arch. Pediatr Cardiol 2006;27:316-20.

66. Dasari TW, Paliotta M. Cervical Aortic Arch. N Engl J Med 2014;371:e38.

67. Rao DP, Ananthakrishna R, Nanjappa M. Pulsatile neck mass: A rare cause. Ann Pediatr Cardiol 2013;6:95.

68. Rajbanshi BG, Gautam NC, Pradhan S, et al. Complex Cervical Aortic Arch With Hypoplasia: A Simple Solution to a Complex Problem. Ann Thorac Surg 2016;102:e27-9.

69. Momma K, Matsuoka R, Takao A. Aortic arch anomalies associated with chromosome 22q11 deletion (CATCH 22). Pediatr Cardiol 1999;20:97-102.

70. Abdoli S, Tatum JM, Fratello AL, et al. Type B Aortic Arch Interruption in an Adult. Ann Thorac Surg 2016;102:e431-2.

71. Patel DM, Maldjian PD, Lovoulos C. Interrupted aortic arch with post-interruption aneurysm and bicuspid aortic valve in an adult: a case report and literature review. Radiol Case Rep 2015;10:5-8.

72. Dillman JR, Yarram SG, D'Amico AR, et al. Interrupted aortic arch: spectrum of MRI findings. AJR Am J Roentgenol 2008;190:1467-74.

73. Nigro Stimato V, Didier D, Beghetti M, et al. Atresia of the Aortic Arch in 4-Year-Old Child: A Clinical Case Study. Front Pediatr 2015;3:19.

74. Singh S, Hakim FA, Sharma A, et al. Hypoplasia, Pseudocoarctation and Coarctation of the Aorta - A Systematic Review. Heart Lung Circ 2015;24:110-8.

75. Vergales JE, Gangemi JJ, Rhueban KS, et al. Coarctation of the aorta - the current state of surgical and transcatheter therapies. Curr Cardiol Rev 2013;9:211-9.

76. Krieger EV, Stout KK, Grosse-Wortmann L. How to Image Congenital Left Heart Obstruction in Adults. Circ Cardiovasc Imaging 2017;10:e004271.

Cite this article as: Priya S, Thomas R, Nagpal P, Sharma A, Steigner M. Congenital anomalies of the aortic arch. Cardiovasc Diagn Ther 2018;8(Suppl 1):S26-S44. doi: 10.21037/ cdt.2017.10.15
77. Chen SS, Dimopoulos K, Alonso-Gonzalez R, et al. Prevalence and prognostic implication of restenosis or dilatation at the aortic coarctation repair site assessed by cardiovascular MRI in adult patients late after coarctation repair. Int J Cardiol 2014;173:209-15.

78. Dijkema EJ, Leiner T, Grotenhuis HB. Diagnosis, imaging and clinical management of aortic coarctation. Heart 2017;103:1148-55.

79. Kitabayashi K, Sakaki M, Araki K, et al. Thoracic aortic aneurysm with aortic pseudocoarctation involving the left subclavian artery. Ann Vasc Surg 2013;27:802.e5-7.

80. Adaletli I, Kurugoglu S, Davutoglu V, et al. Pseudocoarctation. Can J Cardiol 2007;23:675-6.

81. Grigsby JL, Galbraith T, Shurmur S, et al. Pseudocoarctation of the aorta complicated by saccular aneurysm: treatment by aortic arch replacement. Am Heart J 1996;131:200-2.

82. Kim HK, Gottliebson W, Hor K, et al. Cardiovascular anomalies in Turner syndrome: spectrum, prevalence, and cardiac MRI findings in a pediatric and young adult population. AJR Am J Roentgenol 2011;196:454-60.

83. Van Praagh R, Van Praagh S. Persistent fifth arterial arch in man. Congenital double-lumen aortic arch. Am J Cardiol 1969;24:279-82.

84. Khan S, Nihill MR. Clinical presentation of persistent 5 th aortic arch: 3 new cases. Tex Heart Inst J 2006;33:361-4.

85. Uysal F, Bostan OM, Cil E. Coarctation of Persistent 5th Aortic Arch: First Report of Catheter-Based Intervention. Tex Heart Inst J 2014;41:411-3.

86. Hwang MS, Chang YS, Chu JJ, et al. Isolated persistent fifth aortic arch with systemic-to-pulmonary arterial connection. J Thorac Cardiovasc Surg 2003;126:1643-4.

87. Gupta SK, Gulati GS, Anderson RH. Clarifying the anatomy of the fifth arch artery. Ann Pediatr Cardiol 2016;9:62-7.

88. Newman B, Hanneman K, Chan F. Persistent fifth arch anomalies - broadening the spectrum to include a variation of double aortic arch vascular ring. Pediatr Radiol 2016;46:1866-72. 